\title{
Evaluation of Effectiveness of Comprehensive Oral Hygiene Management in Mechanical Ventilation Patients in Chinese Intensive Care Unit Kang Longfei ${ }^{1,2}$, Shinobu Okada ${ }^{{ }^{*}}$, Toshiko Ogawa ${ }^{1}$, Zhang Yingqi ${ }^{{ }^{*}}$
} ${ }^{1}$ Graduate School of Nursing, Chiba University, Japan

${ }^{2}$ The First Hospital of Hebei Medical University, Emergency Department, China

\section{Abstract}

Objective: To investigate the effect of toothbrushes and oral care solution in oral hygiene management on the prevention of ventilator-associated pneumonia (VAP) and ventilator-associated events (VAE) in the critically ill patients.

Methods: The patients were divided into 5 groups according to the time of admission of ICU. Toothbrush and oral care solution were used as intervention for oral care. By comparing halitosis, dental plaque, oral mucosa status, and secretion bacteriological analysis, the changes of oral status were observed and evaluated, and VAE was evaluated and diagnosed according to the corresponding standards. ICU and hospital length of stay, duration of MV were calculated from the medical records. Bacterial count and VAP pathogens of oral secretion were also evaluated.

Results: The incidence rate of VAE, duration of MV and ICU stays and mortality rate in each group of neurosurgical and general ICU did not show a significant difference. VAE developed later in the intervention group using $0.1 \%$ cetylpyridinium chloride solution. The evaluation of halitosis and dental plaque in general and neurosurgical ICU on the 7 th day after intubation were significantly improved compared with those on admission $(\mathrm{P}<0.05)$. However, the bleeding in the patients diagnosed as cerebral hemorrhage increased in the intervention group. The number of S.pneumoniae in oral secretion of each group was different. The bacterial count of intervention groups in general ICU began to decrease on the third day, while that of control group did not decrease significantly or even increased.

Conclusions: The oral care incorporating toothbrush brushing was effective to reduce the bacterial count in the oral cavity and eliminate halitosis and dental plaque in mechanically ventilated patients.

\section{Introduction}

The oral cavity of patients with orotracheal intubation is in the open state for a long time, and it affects the normal physiological activities of oral cavity including saliva secretion and maintenance of normal mucosa. As a result, microorganisms in the mouth proliferate in large quantities and this causes the complications such as halitosis and oral ulcer, and even ventilator-associated pneumonia (VAP) when oral microorganisms are aspirated. Therefore, oral hygiene management of intubated patients is quite important to prevent these undesirable complications.

In 2010 the Centers for Disease Control and Prevention (CDC) suggested that the use of chlorhexidine gluconate for oral care of mechanically ventilated (MV) patients as one of the preventive and therapeutic measures for VAP [1]. Moreover, many studies have shown that toothbrush scrubbing could effectively remove dental plaque and reduce the rate of oropharyngeal bacterial colonization, thereby decreasing the incidence of VAP [2-5]. However, in Chinese ICU, various kinds of solutions other than chlorhexidine gluconate are used for oral care and toothbrush scrubbing is not incorporated into oral care of MV patients. These conditions seem to be due to the oral care guidelines of the Ministry of Health of China in which the use of sterile saline cotton balls is recommended to scrub the oral cavity. In addition to oral care solution and brushing with a toothbrush, the position during oral care, the appropriate range of cuff pressure and suction of subglottic secretion in the MV patient, all of which are shown to be effective for VAP prevention [6-8], are also not specified in Chinese guideline.

Therefore, it is necessary to introduce comprehensive oral hygiene management including appropriate oral solution, brushing with a toothbrush, positioning during oral care, maintenance of appropriate cuff pressure and suction of subglottic secretion into oral care of MV patients in Chinese ICU. This study aimed to evaluate the effectiveness of comprehensive oral hygiene management especially brushing and oral care solutions in MV patients admitted in Chinese ICU.

\section{Materials and Method}

This study was approved by the Ethics Committee of Chiba University, Graduate School of Nursing (29-125). The trial is registered in the China Registry of Clinical Trials (20180050400).

\section{Research design and subjects}

Research design of this study was a non-randomized controlled trial. MV patients who admitted to the general ICU from June to September 2018 and to the neurosurgical ICU from June to September 2018 of the First Hospital of Hebei Medical University in China were enrolled in the trial.

"Corresponding Author: Prof. Shinobu Okada, Graduate school of nursing, Chiba University, 1-8-1, Inohana, Chuo-ku, Chiba-shi, Chiba, 260-0856, Japan; E-mail: sokada@faculty.chiba-u.jp

*Corresponding Author: Mr. Yingqi Zhang, The First Hospital of Hebei Medical University, emergency department, 89 Donggang road, Shijiazhuang, Hebei 050031, China; E-mail: 276557406@qq.com

Citation: Longfei K, Okada S, Ogawa T, Zhang Y (2021) Evaluation of Effectiveness of Comprehensive Oral Hygiene Management in Mechanical Ventilation Patients in Chinese Intensive Care Unit. Int J Nurs Clin Pract 8: 346. doi: https://doi. org/10.15344/2394-4978/2021/346

Copyright: (C) 2021 Longfei et al. This is an open-access article distributed under the terms of the Creative Commons Attribution License, which permits unrestricted use, distribution, and reproduction in any medium, provided the original author and source are credited. 
The inclusion criterion were $>18$ years old and undergoing MV for $>48$ hours. The exclusion criteria were previous history of pneumonia, pregnancy, suffering from oral trauma requiring special oral care, allergic to oral care solutions, and having autoimmune diseases.

\section{Comprehensive oral hygiene management}

In the general ICU, the patients were divided into the control group $\mathrm{G}$, intervention group A and B depending on the date of ICU admission (Control group: from June 1 to 30 . Intervention group $A$ from July 1 to August 15; Intervention group B: from August 16 to September 30).

Patients in the control group $\mathrm{G}$ received conventional oral care of the general ICU (swabbing with cotton ball containing $0.1 \%$ cetylpyridinium chloride solution (CPC) at 8 and 20 o'clock daily); the intervention group A was given oral brushing with a toothbrush on the basis of routine oral care; in the intervention group $\mathrm{B}, 0.1 \%$ cetylpyridinium chloride solution (CHG) was replaced to $0.2 \%$ chlorhexidine gluconate solution, and others were the same as those in intervention group A.

In the neurosurgical ICU, the patients were divided into control group $\mathrm{N}$ and intervention group $\mathrm{C}$ depending on the date of ICU admission (Control group: from June 1 to July 30 . Intervention group C: from August 1 to September 30).

In the control group $\mathrm{N}$, conventional oral care method of the neurosurgical ICU (scrub with sterile saline cotton balls at 8 and 20 o'clock daily) was provided; in the intervention group $\mathrm{C}$, toothbrush scrubbing was added to conventional oral care method.

For brushing, a toothbrush with small head, which is convenient for oral care of patients with orotracheal intubation was used in both ICUs.

In addition to oral care in each group mentioned above, all patients received standard preventive measures for VAP prophylaxis These included semi-recumbent position, hand washing, suctioning and drainage of subglottic secretions when needed, using heat and moisture exchanger filter, and changing of tubes or ventilator circuit only when needed. The cuff pressure was maintained between 20 and 30 $\mathrm{mmHg}$ in all groups. Antibiotic therapy was conducted when needed.

\section{Data Collection}

\section{Demographic characteristics}

Age, sex, medical history, and acute physiology and chronic health evaluation score (APACHE II ) at the ICU admission were obtained from the hospital record for all patients.

\section{Evaluation of comprehensive oral hygiene management}

The primary outcome was the occurrence of VAP and VAE, and secondary outcomes included ICU and hospital length of stay, duration of MV, bacterial count and detection of VAP pathogens of oral secretion, and oral conditions of the patients.

\section{VAE surveillance}

Positive end-expiratory pressure (PEEP), and fraction of inspiratory oxygen $\left(\mathrm{FiO}_{2}\right)$ were recorded using VAE surveillance sheet several times per day during MV. Ventilator-associated condition (VAC), infection-related ventilator-associated complications (IVAC), possible or probable pneumonia and VAP were defined according to CDC's VAE surveillance algorithm. Administered antibiotics, laboratory reports on isolated bacteria and records of body temperature were also obtained to confirm the judgement.

\section{Duration of MV and ICU stay and mortality}

Duration of MV and ICU stay, and mortality were calculated from the medical records.

\section{Bacterial count and detection of VAP pathogen of oral secretion}

The secretions above the cuff were collected at the subglottic suction before oral care approximately 48 hours after tracheal intubation (the second day of MV). The secretions were also collected on the third and seventh day of MV. The aspirate was then homogenized by mixing with three times the amount of sputum dissolving enzyme solution (Sputazyme, Kyokuto pharmaceutical Co., Ltd, Japan). The bacterial count of homogenized aspirate was determined using a bacterium counting device (bacterial counter Panasonic dental health care, Japan). After the total bacterial count was determined, $100 \mu \mathrm{l}$ homogenized aspirate were diluted as needed and inoculated into four kinds of VAP pathogen selection media (NAC agar medium for P.aerugianosa; mannitol salt agar medium for S.aureus sheep blood agar M70: for S.pneumoniae; multidrug-resistant Acinetobacter selective medium: for A.baumannii). After the cultivation at $37^{\circ} \mathrm{C}$ for 24 hours, each VAP pathogen was identified according to the colony appearance on the selective medium and was counted. Then, identified VAP pathogens were biochemically confirmed using an automated bacteria identification system (Microbial ID/AST System DL-96, Zhuhai DL Biotech CO., Ltd, China).

\section{Assessment of oral condition}

The patient's oral condition was assessed using the BRUSHED Teeth oral condition assessment tool [9] by the researcher on the first, second, and seventh days of ICU stay. When the patient was extubated or discharged the ICU before the seventh day, it was assessed on the day of extubation or the day of ICU discharge. In BRUSHED Teeth oral condition assessment tool, bleeding, redness, ulceration, saliva, halitosis, external factors, plaque, and teeth are scored from 0 to 2 (for halitosis, external factors, plaque, and teeth) or 3 (for bleeding, redness, ulceration). Scores of bleeding, halitosis, plaque and total score between the second day and the seventh day, (the day of extubation when the patient was extubated or the day of ICU discharge when the patient discharged the ICU before the seventh day) were compared. For total and each score of oral condition was categorized as "improved", "unchanged", or "worsened" when the score decreased, unchanged or increased, respectively. For bleeding, halitosis, and plaque, it was assessed as "improved or without bleeding/halitosis/ plaque" when the score of the seventh day or the day of extubation was 0 , whereas it was evaluated to be "with bleeding/halitosis/plaque" when the score was 1or more.

\section{Statistical Analysis}

Values were expressed as mean $\pm \mathrm{SD}$ when they were distributed normally or median and range were used when they did not follow normal distribution. Student's t-test or one-way ANOVA analysis was used for the comparison of continuous variables. Chi-square test was 
Citation: Longfei K, Okada S, Ogawa T, Zhang Y (2021) Evaluation of Effectiveness of Comprehensive Oral Hygiene Management in Mechanical Ventilation Patients in Chinese Intensive Care Unit. Int J Nurs Clin Pract 8: 346. doi: https://doi.org/10.15344/2394-4978/2021/346

Page 3 of 6

used for the comparison of categorical data. All statistical analyses were carried out with statistical package for the social sciences software version 24.0 (SPSS, Chicago, IL, USA). For all analyses, P values less than 0.05 were considered significant.

\section{Results}

\section{Participants}

A total of 57 patients, 34 in general ICU and 23 in neurosurgical ICU, were included in the analysis (Figure 1). Table 1 shows the demographic characteristics of the patients. There were no significant statistical differences among the control group $\mathrm{G}$, intervention group $\mathrm{A}$ and intervention group B of the general ICU, and between the control group $\mathrm{N}$ and intervention group $\mathrm{C}$ of the neurosurgical ICU.

\section{VAE surveillance, duration of MV days, ICU stay and mortality}

Table 2 shows the summary of VAE morbidity, the duration of $\mathrm{MV}$, ICU stay and mortality. VAE morbidity in the general ICU were
$2 / 10(20.0 \%), 2 / 15(13.3 \%)$, and $2 / 9(22.2 \%)$ in the control group $\mathrm{G}$, intervention group $\mathrm{A}$, and the intervention group $\mathrm{B}$, respectively. In the neurosurgical ICU, they were $2 / 11(18.2 \%)$ in the control group $\mathrm{N}$ and $2 / 12(16.7 \%)$ in the intervention group $C$. There were no significant differences among groups both in the general and neurosurgical ICU. There were 2 cases of PVAP. One case of control group G developed PVAP on the third day after intubation, and S.aureus, was determined as a causative bacterium. The other case in the control group $\mathrm{N}$ also developed PVAP on the seventh day after intubation, and A.baumannii was determined as a causative bacterium.

There is no significant difference among the three groups of general ICU and between the groups of neurosurgical ICU. In addition, the cause of death was not related to VAE.

\section{Changes in oral condition}

Figure 2 shows the changes of halitosis, plaque, and bleeding scores from the second to the seventh day (or the day leaving the ICU or the day of extubation). For the general ICU, the score of halitosis and
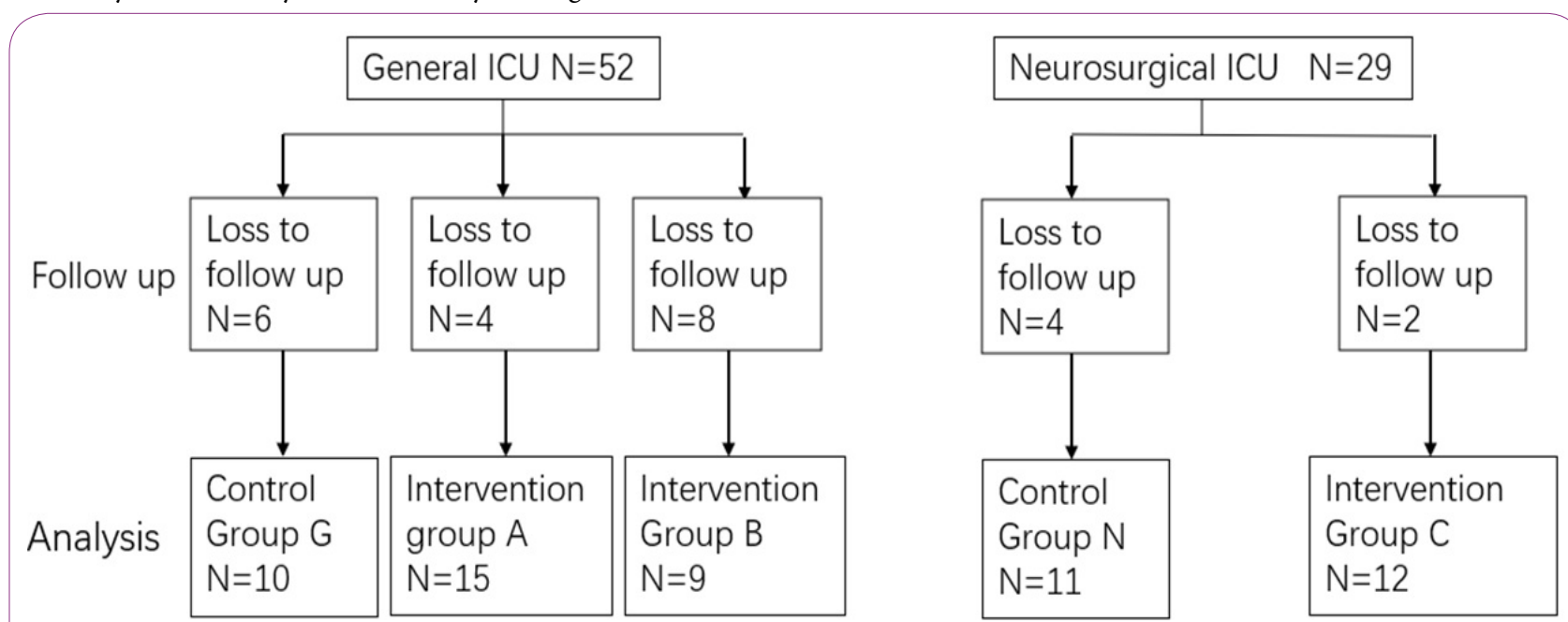

Figure 1: Study overview.

Table 1: Baseline Demographics and Clinical Information of the patient.

\begin{tabular}{|l|l|l|l|l|l|}
\hline & \multicolumn{3}{|c|}{ General ICU } & \multicolumn{2}{c|}{ Neurosurgical ICU } \\
\hline Patient data & Control G (N=10) & Intervention A (N=15) & Intervention B (N=9) & Control N (N=11) & Intervention C (N=12) \\
\hline Age, mean \pm SD (y) & $56.8 \pm 15.2$ & $48.5 \pm 20.1$ & $62 \pm 14.6$ & $61.2 \pm 12.9$ & $55.8 \pm 18.4$ \\
\hline Male, $\mathrm{n}(\%)$ & $8(80)$ & $11(73.3)$ & $5(55.6)$ & $6(54.5)$ & $4(33.3)$ \\
\hline Admission diagnosis, $\mathrm{n}(\%)$ & & & & & \\
\hline Cardiac arrest & 5 & 4 & 0 & & \\
\hline Ischemic heart disease & 1 & 3 & 4 & & \\
\hline Respiratory failure & 0 & 3 & 1 & & \\
\hline Metabolic acidosis & 0 & 1 & 1 & & \\
\hline Infection & 0 & 2 & 0 & & \\
\hline Trauma & 2 & 1 & 3 & & 7 \\
\hline cerebral hemorrhage & & & & 3 & 5 \\
\hline cerebral infarction & & & 0 & & \\
\hline Other & 2 & 1 & $28.7 \pm 4.6$ & $22.3 \pm 4.0$ & $23.3 \pm 4.1$ \\
\hline APACHE II, mean \pm SD & $26.9 \pm 6.0$ & $27.1 \pm 6.9$ & & & \\
\hline APAC & & & & & \\
\hline
\end{tabular}

APACHE II: Acute physiology and Chronic Health Evaluation II. 
Citation: Longfei K, Okada S, Ogawa T, Zhang Y (2021) Evaluation of Effectiveness of Comprehensive Oral Hygiene Management in Mechanical Ventilation Patients in Chinese Intensive Care Unit. Int J Nurs Clin Pract 8: 346. doi: https://doi.org/10.15344/2394-4978/2021/346

Page 4 of 6

plaque on the seventh day after intubation (extubation or withdrawal from ICU) were significantly improved compared with those on the second day after intubation $(\mathrm{p}=0.000, \mathrm{p}=0.022)$. For neurosurgical ICU, halitosis and plaque on the seventh day were also significantly improved $(p=0.001, p=0.002)$. However, the bleeding in intervention group $\mathrm{C}$ increased after the intervention whereas bleeding was not observed in the control group $\mathrm{N}$ and for that reason percentage of the participants showing improvement of total score in the intervention group $\mathrm{C}$ was similar to that of the control group N. In addition, in the neurosurgical ICU, the patients who were found with oral bleeding, were all diagnosed with cerebral hemorrhage.

\section{Bacterial count of oral secretion}

Figure $3 \mathrm{~A}$ shows the changes in the bacterial count of the oral secretions. In the general ICU, bacterial count in the intervention group A and B decreased on the seventh day while it continuously increased in the control group $\mathrm{G}$. The median bacterial count of the intervention group A and B were about half of the control group $G$ although there were no statistical differences. In the neurosurgical ICU, the bacterial count tended to decrease both in the control group $\mathrm{N}$ and intervention group $\mathrm{C}$, and the reduction of bacterial count in the intervention group was smaller than that in the control group. As a result, the bacterial count in the intervention group was significantly higher than that in the control group on the seventh day $(\mathrm{p}=0.032)$.

Figure 3B shows the changes in the number of S.pneumoniae in individual patients's oral secretions of each group. In general ICU, the bacterial count in intervention group A and B began to decrease on the day 3, while the decrease in control group $\mathrm{G}$ was not obvious or even increased. In the neurosurgical ICU, the bacterial count began to decline on the day 7 in 6 of 9 subjects of the intervention

\begin{tabular}{l} 
Table 2: Summary of VAE surveillance, and MV, ICU stay days and mortality. \\
\begin{tabular}{|l|l|l|l|l|l|}
\hline \multirow{3}{*}{} & \multicolumn{3}{|c|}{ General ICU } & \multicolumn{3}{c|}{ Neurosuigical ICU } \\
\cline { 2 - 6 } & Control G & Intervention A & Intervention B & Control N & Intervention C \\
\cline { 2 - 6 } & $(\mathrm{N}=10)$ & $(\mathrm{N}=15)$ & $(\mathrm{N}=9)$ & $(\mathrm{N}=11)$ & $(\mathrm{N}=12)$ \\
\hline VAE morbidity & $2(20 \%)$ & $2(13.3 \%)$ & $2(22.2 \%)$ & $2(18.2 \%)$ & $2(16.7 \%)$ \\
\hline VAE onset days & day4(VAC) & day3(VAC $)$ & day7(VAC $)$ & day7(PVAP) & day5 (VAC) \\
\cline { 2 - 6 } & day3(PVAP) & day3(IVAC) & day11(VAC) & day7(IVAC) & day5(IVAC) \\
\hline MV days(median, range) & $6(3 \sim 72)$ & $6(3 \sim 22)$ & $6(4 \sim 11)$ & $9(3 \sim 17)$ & $10(3 \sim 16)$ \\
\hline ICU stay(median, range) & $7(3 \sim 72)$ & $11(4 \sim 22)$ & $7(4 \sim 11)$ & $7(5 \sim 17)$ & $7(4 \sim 17)$ \\
\hline mortality(\%) & $2(20)$ & 0 & $2(13.3)$ & $2(18.2)$ & $1(8.3)$ \\
\hline
\end{tabular} \\
\hline
\end{tabular}

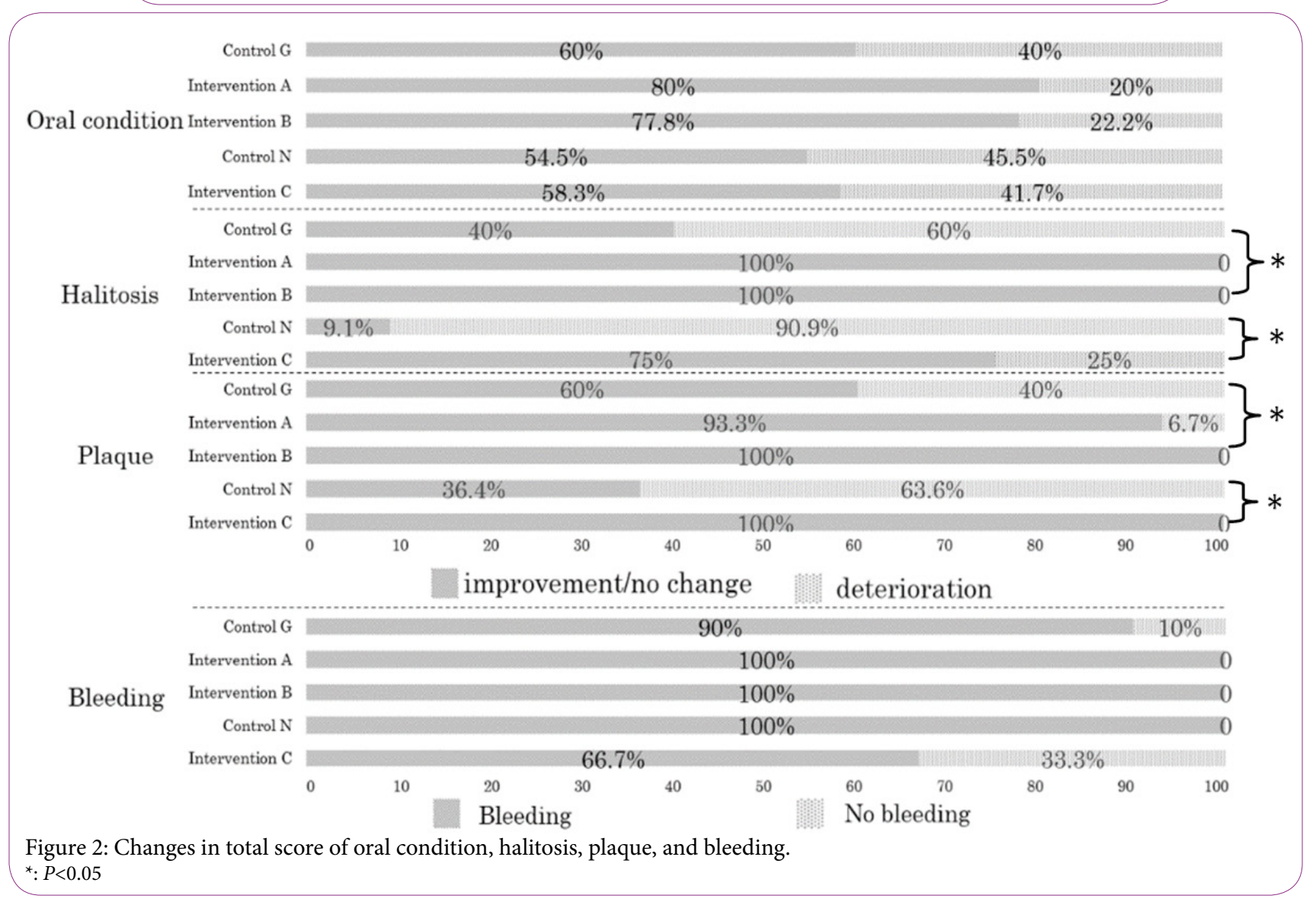


Citation: Longfei K, Okada S, Ogawa T, Zhang Y (2021) Evaluation of Effectiveness of Comprehensive Oral Hygiene Management in Mechanical Ventilation Patients in Chinese Intensive Care Unit. Int J Nurs Clin Pract 8: 346. doi: https://doi.org/10.15344/2394-4978/2021/346

groups, although data on the seventh day was obtained from only one subject in the control group. Comparison was not done for VAP pathogens other than S.pneumoniae due to the small number of patients in whom the pathogen were detected.

\section{Discussion}

Although the incidence of VAE did not differ among the control and the intervention groups both in the general and neurosurgical ICUs, the PVAP was not observed in the intervention groups. There was no significant difference between ICU stay and MV days.

Judging from the incidence of VAE, the effect of CPC was similar to $\mathrm{CHG}$, and the effect of brushing was not obvious. However, PVAP occurred only in the control group in which brushing with toothbrush was not included in the oral care.

On the other hand, from the day of VAE onset, VAE occurred within one week in the control group $\mathrm{G}$ and the intervention group A using CPC, while it occurred one week after intubation in the intervention group B using CHG. It has been reported that $\mathrm{CHG}$ has a long-lasting bactericidal effect and is effective against Gramnegative bacilli that cause late-onset VAP [10]. Therefore, CHG could inhibit the proliferation both of early and late VAP pathogens in oral secretions and this may be related to delayed onset of VAE in the intervention group $\mathrm{B}$.

Antacids routinely used for prevention of stress ulcers is considered to change the pathogen in late-onset VAP. Increase in $\mathrm{pH}$ of gastric juice allows the excessive reproduction of Gram-negative bacteria, thereby colonizing the stomach. Stomach contents containing Gramnegative bacilli easily regurgitate and flow into the respiratory tract because of weakened swallowing/cough reflexes in patients with tracheal intubation. CPC is a quaternary ammonium compound with a pyridine ring. It has a strong bactericidal and growth inhibitory effect on Gram-positive bacteria such as staphylococcus. But its inhibitory effect on Gram-negative bacteria is poorer than that against Gram-positive bacteria [11]. On the contrary CHG, which belongs to a cationic surfactant, a kind of broad-spectrum bactericide has a bactericidal effect on most Gram-positive and Gram-negative bacteria. It adsorbs on the surface of bacterial cell membranes and exerts antibacterial effects, promotes cytoplasmic outflow, and inhibits cell wall repair. Therefore, it is suggested CHG is ideal, as a mouthwash for prevention of late-onset VAP.

The total bacterial count of intervention group A and B in the general ICU using toothbrush brushing gradually decreased although no significant difference was found compared with the control group. It is suggested that toothbrush scrubbing could remove dental plaque through the physical friction of the toothbrush and reduce the number of bacteria including VAP pathogen in the oral cavity, thereby inhibiting the development of PVAP in the intervention group A and B. It is also suggested that bactericidal oral solution is not enough to reduce the oral bacteria and combination with toothbrushing is required. Zanatta et al. reported that $\mathrm{CHG}$ need to be combined with the mechanical brushing with the toothbrush to destroy the bacterial biofilm because the biofilm disturb the penetration of CHG and affect its antibacterial effect [10].

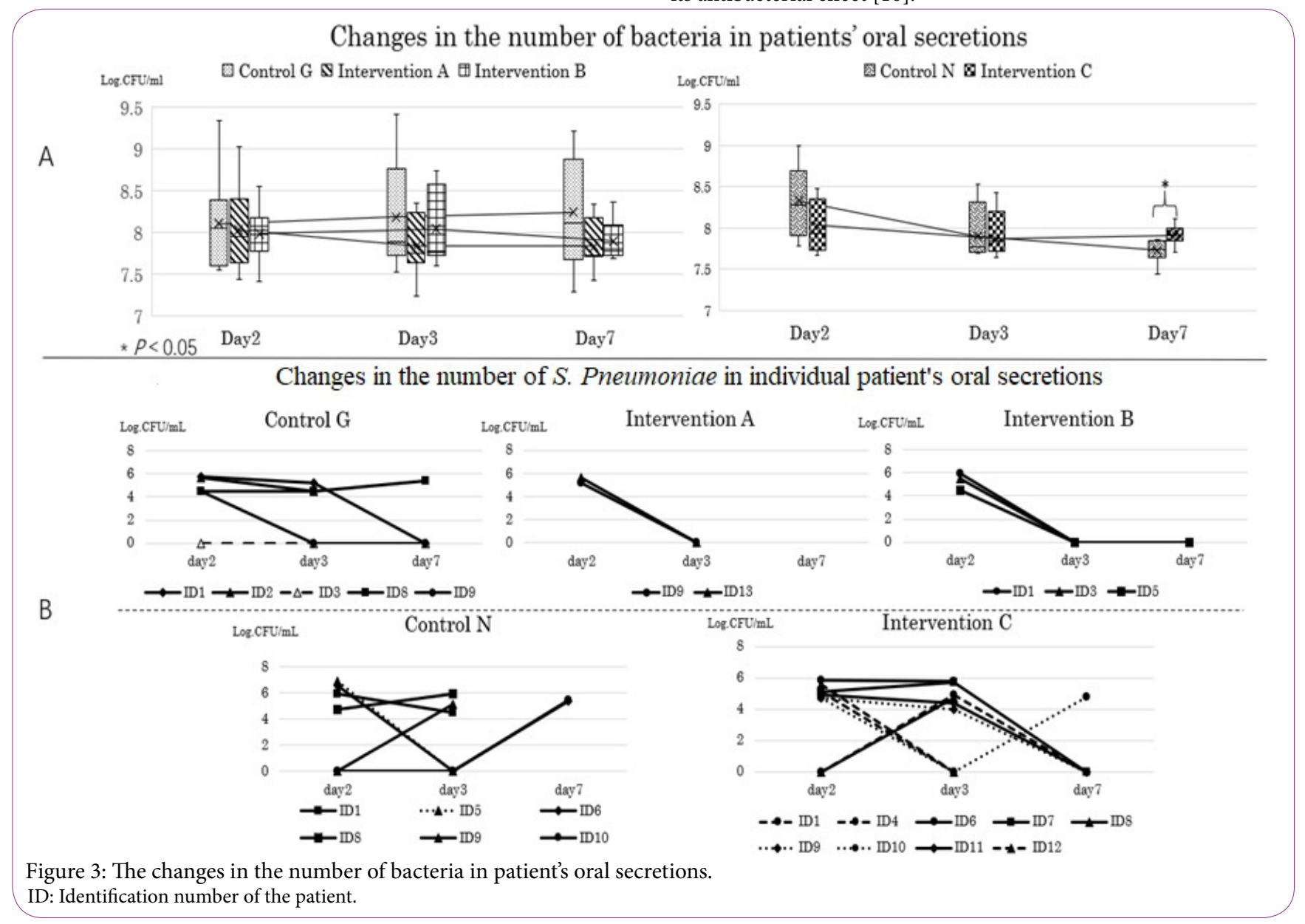


Oral condition, including total BRUSHED scores, halitosis and plaque scores improved in all intervention groups compared to the second day except bleeding of the intervention group C. It can be speculated that brushing could eliminate plaque and reduce halitosis, which is consistent with the report of Stevens et al. [12].

However, contrary to the improvement in halitosis and plaque score, the bleeding score in intervention group $\mathrm{C}$ in the neurosurgical ICU increased after the intervention. It is considered due to the underlying disease of the patients in the neurosurgical ICU. All subjects who showed gingival bleeding in the neurosurgical ICU were diagnosed with cerebral hemorrhage. For patients with intracerebral hemorrhage, repeated use of mannitol to prevent brain edema may lead to dry oral mucosa in patients with endotracheal intubation due to dehydration. Dried mucosa is more vulnerable to mechanical stimuli and physical friction of the toothbrush might damage the dried oral mucosa, resulting in bleeding because the toothbrush used in the study was an ordinary toothbrush except having small head. The damage of oral mucosa would provide a good environment for the proliferation of oral bacteria, and this might cause a significantly higher bacterial count in the intervention group $\mathrm{C}$ on the seventh day after intubation. Therefore, a soft bristle toothbrush or silicone toothbrush should be selected for oral care of the patients prone to oral mucosal bleeding, and the oral state of patients with endotracheal intubation should be carefully observed at any time. An attention to keep oral mucosa moist should be also paid for those patients, especially undergoing repeated mannitol administration.

\section{Conclusions}

The present study showed that the oral care incorporating toothbrush brushing was effective to reduce the bacterial count in the oral cavity and to eliminate halitosis and dental plaque in mechanically ventilated patients. CHG seemed to be more effective for the prevention of late-onset VAP because of its broad bactericidal activity including gram-negative bacteria. In addition, for patients with vulnerable oral mucosa, it is necessary to pay attention to the choice of oral care tools for brushing and to observe the oral mucosa carefully.

\section{Limitation}

Due to the small number of research subjects, the effect of intervention measures on the incidence of VAE was not found.

\section{Author Contribution}

Study conception and design: SO\& ZY

Data collection: LK

Data analysis and interpretation: LK \& SO

Drafting of the article: LK\& TO

Critical revision of the article: All authors.

\section{Competing Interests}

The authors declare that they have no competing interests.

\section{References}

1. Tablan OC, Anderson L, Besser R, Bridges C, Hajjeh R, et al. (2014) Guidelines for preventing health-care--associated pneumonia, 2003: recommendations of $C D C$ and the Healthcare Infection Control Practices Advisory Committee. MMWR Recomm Rep 53: 1-36.
2. Needleman IG, Hirsch NP, Leemans M, Moles DR, Wilson M, et al. (2011) Randomized controlled trial of toothbrushing to reduce ventilatorassociated pneumonia pathogens and dental plaque in a critical care unit. $J$ Clin Periodontol 38: 246-252.

3. Moustafa MF, Tantawey NM (2016) The Effect of Oral Care Intervention on the Occurrence of Ventilator-associated Pneumonia. Gynecology \& Obstetrics 6: 95-100.

4. Vidal C, Vidal A, Monteiro J, Cavalcanti A, Henriques A, et al. (2017) Impact of oral hygiene involving toothbrushing versus chlorhexidine in the prevention of ventilator-associated pneumonia: a randomized study. BMC Infect Dis 17: 173

5. Camargo LD, Silva S, Chambrone L (2019) Efficacy of toothbrushing procedures performed in intensive care units in reducing the risk of ventilator-associated pneumonia: A systematic review. J Periodontal Res 54: 601-611.

6. Mao Z, Gao L, Wang G, Liu C, Zhao Y, et al. (2016) Subglottic secretion suction for preventing ventilator-associated pneumonia: an updated metaanalysis and trial sequential analysis. Crit Care 20: 1-14.

7. Burja S, Belec T, Bizjak N, Mori J, Markota A, et al. (2018) Efficacy of a bundle approach in preventing the incidence of ventilator associated pneumonia (VAP). Bosn J Basic Med Sci 18: 105-109.

8. Jie LI, Ying XU, Jiang J, Icu DO (2019) Continuous control of tracheal cuff pressure and subglottic secretion drainage for preventing ventilatorassociated pneumonia. Anhui Medical and Pharmaceutical Journal 23: 1838-1841.

9. Miaomiao W, Meizhen Z, Tieying Z (2015) Oral Care Assessment Scale for Orally Intubated Critically Ill Patients: making and testing of reliability and validity. Journal of Nrusing Science 30: 5-11.

10. Zanatta FB, Antoniazzi RP, Rösing CK (2007) The Effect of $0.12 \%$ Chlorhexidine Gluconate Rinsing on Previously Plaque-Free and PlaqueCovered Surfaces: A Randomized, Controlled Clinical Trial. J Periodontol 78: 2127-2134

11. Sandt C, Barbeau J, Gagnon MA, Lafleur M (2007) Role of the ammonium group in the diffusion of quaternary ammonium compounds in Streptococcus mutans biofilms. J Antimicrob Chemother 60: 1281-1287.

12. Stevens JP, Silva G, Gillis J, Novack V, Talmor D, et al. (2014) Automated surveillance for ventilator-associated events. Chest 146: 1612-1618. 